\title{
Bell inequality violation with two remote atomic qubits
}

\author{
D. N. Matsukevich $*$ P. Maunz, D. L. Moehring S. Olmschenk, and C. Monroe \\ Department of Physics and Joint Quantum Institute, \\ University of Maryland, College Park, Maryland, 20742
}

(Dated: October 27, 2018)

\begin{abstract}
We observe violation of a Bell inequality between the quantum states of two remote $\mathrm{Yb}^{+}$ions separated by a distance of about one meter with the detection loophole closed. The heralded entanglement of two ions is established via interference and joint detection of two emitted photons, whose polarization is entangled with each ion. The entanglement of remote qubits is also characterized by full quantum state tomography.

PACS numbers: 03.65.Ud, 03.67.Mn, 37.10.Ty, 42.50.Xa
\end{abstract}

In 1964 Bell showed that in all local realistic theories, correlations between the outcomes of measurements in different parts of a physical system satisfy a certain class of inequalities [1]. Furthermore, he found that some predictions of quantum mechanics violate these inequalities. Starting with the first experimental tests of Bell inequalities with photons [2, 3, 4], violation of a Bell inequality has been observed in a wide range of systems including protons [5], K-mesons [6], ions [7], neutrons [8], Bmesons [9], heterogeneous atom-photon systems [10, 11] and atomic ensembles 12, 13. Demonstration of the violation of a Bell inequality has also become a routine technique to verify the presence of entanglement and check the security of a quantum communication link [14].

In order to exclude all local realistic theories, a rigorous experimental test of a Bell inequality must satisfy two conditions. First, the measurement time has to be sufficiently short such that no information traveling at the speed of light can propagate from one qubit to another during the measurement (locality loophole). Second, the efficiency of the quantum state detection has to be high enough such that it is impossible to mimic a Bell inequality violation by selective choice of the successful measurement events (detection loophole). Since photons can propagate over a long distance and be detected fast, the locality loophole was first closed in a photon system [3, 4]. On the other hand, high detection efficiency and deterministic preparation of an entangled state of trapped ions has closed the detection loophole in a system of two trapped ions separated by $\sim 3 \mu \mathrm{m}$ [7]. Although several experimental schemes for a loophole-free Bell inequality test have been proposed [15, 16, 17, 18, 19], no experiment to date has closed both loopholes simultaneously.

One of these proposals (by Simon and Irvine [18]) combines the advantages of photons and trapped ions. This protocol starts by preparing two spatially separated ions, each entangled with its emitted photon. These photons are then sent to an intermediate location where a partial Bell state analysis is performed. Successful detection of an entangled state of two photons unambiguously heralds the preparation of an entangled state of the two ions. The
Bell inequality violation is then verified by local rotation and detection of the ion qubits [7].

In this Letter, we report an important step towards implementation of this protocol with the observation of a Bell inequality violation using two ${ }^{171} \mathrm{Yb}^{+}$ions separated by about 1 meter. In contrast to our previous work where the photonic qubit was encoded in the frequencies of a photon [20, 21], here we use the polarization degree of freedom for the photonic qubit and two nearly degenerate states of the atom to encode the atomic qubit. This allows for measurement of both atomic and photonic qubits in arbitrary bases and for characterization of the generated ion-photon and ion-ion entangled states by quantum state tomography, resulting in an ion-ion entanglement fidelity of $81 \%$. Together with the high efficiency of detecting the quantum state of an ion, such a fidelity makes it possible to observe a Bell inequality violation between two distant particles with the detection loophole closed. With an even larger separation between the ions or faster detection of the atomic qubit, the technique demonstrated here may ultimately allow for a loophole-free Bell inequality test [18, 22].

The experimental setup is shown in Fig. 1. A single ${ }^{171} \mathrm{Yb}^{+}$ion is stored in each of two rf-Paul traps located in independent vacuum chambers. The ions are placed in a magnetic field of $4.6 \mathrm{G}$ parallel to the direction of the quantization axis. A $700 \mathrm{~ns}$ light pulse at $369.5 \mathrm{~nm}$ resonant with the ${ }^{2} S_{1 / 2}, F=1 \rightarrow{ }^{2} P_{1 / 2}, F=1$ transition optically pumps the ions in both traps to the ${ }^{2} S_{1 / 2}, F=0, m_{F}=0\left({ }^{2} S_{1 / 2}|0,0\rangle\right)$ state. The ${ }^{2} P_{1 / 2}$ state has a probability of $\simeq 0.005$ to decay to the metastable ${ }^{2} D_{3 / 2}$ state. To prevent population trapping in this state, the ion is illuminated with $935.2 \mathrm{~nm}$ light resonant with the ${ }^{2} D_{3 / 2} \rightarrow{ }^{3} D[3 / 2]_{1 / 2}$ transition [23]. After optical pumping, $\mathrm{a} \simeq 2$ ps light pulse from a frequency doubled, mode-locked, Ti-sapphire laser, polarized linearly along the direction of the magnetic field, transfers the population to the excited ${ }^{2} P_{1 / 2}|1,0\rangle$ state with near unit efficiency. Since the duration of the excitation pulse is much shorter than the excited state lifetime $(\simeq 8 \mathrm{~ns})$, at most one $369.5 \mathrm{~nm}$ photon can be emitted by the ion 24]. The duration of each optical pumping and excitation cycle is 

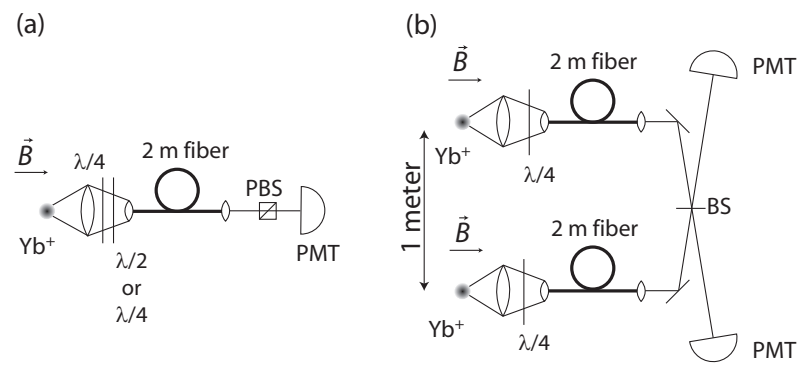

FIG. 1: Experimental setup for (a) the ion-photon and (b) ion-ion experiments. $\vec{B}$ shows the direction of applied magnetic field. PBS: polarizing beamsplitter; PMT: photomultiplier tube; BS: 50/50 nonpolarizing beamsplitter; $\lambda / 2$ : halfwave plate; $\lambda / 4$ : quarter-wave plate.

$1.4 \mu s$. After 107 cycles, the ions are Doppler cooled for $40 \mu s$. The average overall excitation rate is $0.52 \mathrm{MHz}$.

When viewed along the quantization axis, the ${ }^{2} P_{1 / 2}|1,0\rangle$ state either decays to the ${ }^{2} S_{1 / 2}|1,1\rangle$ state emitting a left circular $\left(\sigma^{-}\right)$polarized photon or to the ${ }^{2} S_{1 / 2}|1,-1\rangle$ state emitting a right circular $\left(\sigma^{+}\right)$polarized photon. According to the dipole radiation pattern, $\pi$ polarized photons emitted due to decay to the ${ }^{2} S_{1 / 2}|0,0\rangle$ state cannot propagate along the quantization axis direction. Therefore, when the photon is emitted along the quantization axis, the state of each ion is entangled with the polarization state of its emitted photon:

$$
\Psi=\frac{1}{\sqrt{2}}\left(|1,1\rangle\left|\sigma^{-}\right\rangle-|1,-1\rangle\left|\sigma^{+}\right\rangle\right) .
$$

For each ion, the emitted photons are collected by an imaging lens (numerical aperture 0.23) and sent through a $\lambda / 4$ wave plate to convert $\sigma^{+}$or $\sigma^{-}$circular polarization to linear horizontal $(H)$ or vertical $(V)$ polarization, respectively. The state of each ion-photon system given the photon passes the quarter-wave plate can be written as

$$
\Psi=\frac{1}{\sqrt{2}}(|1,1\rangle|V\rangle-i|1,-1\rangle|H\rangle)
$$

The photons from each ion are coupled to a single mode fiber to facilitate mode-matching on a nonpolarizing 50/50 beamsplitter. Photons at the output ports of the beamsplitter are detected with photomultiplier tubes (PMTs) (see Fig. 1(b)). The contrast of interference between two modes is $97 \%$, the quantum efficiency of each PMT is about $15 \%$, and the count rate due to the dark counts and background light leakage is about $3 \mathrm{~Hz}$. The arrival times of the photo-electric pulses from the PMTs are recorded by a time to digital converter. Coincidence detection of two photons interrupts an experimental time sequence and triggers a sequence of microwave pulses to perform rotation of the ion qubits, followed by state detection of the ions using standard fluorescence techniques [23].

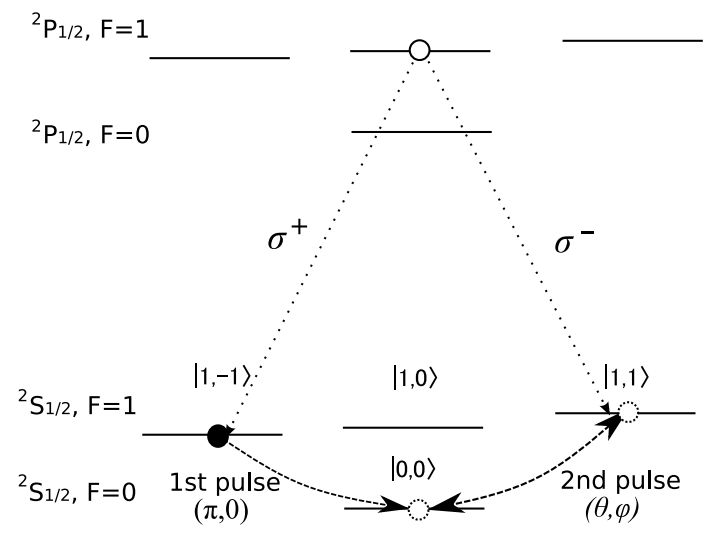

FIG. 2: The ion-photon entanglement scheme and sequence of microwave pulses for ion qubit manipulations. $\sigma^{+}$and $\sigma^{-}$ are right and left circular polarization of a photon, $\theta$ is proportional to the duration of the second microwave pulse and $\phi$ is a relative phase between first and second microwave pulses.

Given perfect mode-matching of the input single photon wavepackets on the beamsplitter, detection of a photon at each output port of the beamsplitter corresponds to a successful measurement of photons in the state [25]

$$
\Psi_{p h}=\frac{1}{\sqrt{2}}(|H\rangle|V\rangle-|V\rangle|H\rangle) .
$$

This projects ions $(a)$ and $(b)$ onto the entangled state [18, 21]

$$
\Psi_{i o n}=\frac{1}{\sqrt{2}}\left(|1,1\rangle_{a}|1,-1\rangle_{b}-|1,-1\rangle_{a}|1,1\rangle_{b}\right) .
$$

Due to the Zeeman splitting of the ground states of the ion, the emitted $\sigma^{+}$and $\sigma^{-}$polarized photons have a frequency difference of about $13 \mathrm{MHz}$. Nevertheless, it is still possible to get the entangled state of Eq.(4) if the photons with the same polarization also have the same frequency.

Quantum state tomography of the atomic qubits requires the ability to detect the state of each ion in an arbitrary basis. For this we first apply a resonant microwave $\pi$ pulse to transfer the population from ${ }^{2} S_{1 / 2}|1,-1\rangle$ to the ${ }^{2} S_{1 / 2}|0,0\rangle$ state. Next, a second microwave pulse resonant with the ${ }^{2} S_{1 / 2}|0,0\rangle \leftrightarrow{ }^{2} S_{1 / 2}|1,1\rangle$ transition, with a controlled duration and phase relative to the first pulse, is applied to perform a qubit rotation (see Fig. 2). As a result, the state of the ion is transformed as follows:

$$
\begin{aligned}
\cos \left(\theta_{i} / 2\right)|1,1\rangle+\sin \left(\theta_{i} / 2\right) e^{i \phi}|1,-1\rangle & \rightarrow|1,1\rangle \\
-\sin \left(\theta_{i} / 2\right) e^{-i \phi}|1,1\rangle+\cos \left(\theta_{i} / 2\right)|1,-1\rangle & \rightarrow|0,0\rangle .
\end{aligned}
$$

Here $\theta_{i}$ is proportional to the duration of the second microwave pulse, and $\phi$ is the relative phase between the first and second pulses [10, 20, 26].

The fluctuations of the magnetic field at the position of the ion can change the phase that each ion acquires 

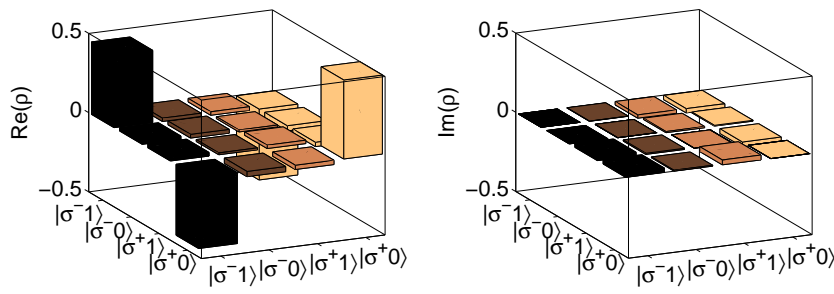

FIG. 3: Real (left) and imaginary (right) parts of reconstructed density matrix for the system of a single photon and an ion. Results are based on 42495 events.

before state detection. To keep the magnitude of the magnetic field constant, the experimental sequence is interrupted about 20 times a second to perform a Ramsey experiment. The ions in each trap are first optically pumped to the $|0,0\rangle$ ground state. Two microwave $\pi / 2$ pulses resonant with the $|0,0\rangle \leftrightarrow|1,-1\rangle$ transition separated by $200 \mu s$ are applied and then the state of each ion is detected. The probability to find the ion in the $|1,-1\rangle$ state is continuously monitored and the current in the bias coil is adjusted to keep the magnetic field magnitude constant. We estimate that fluctuations of the magnetic field do not exceed $1 \mathrm{mG}$ over the several days of experiment.

Following microwave rotations, the state of the $\mathrm{Yb}^{+}$ ion is detected. The $369.5 \mathrm{~nm}$ light resonant with the ${ }^{2} S_{1 / 2}, F=1 \rightarrow{ }^{2} P_{1 / 2}, F=0$ transition impinges on the ion and the fluorescence is detected with a PMT. Ideally, if an ion is in the $F=1$ state, it scatters this light. On the other hand, if the ion is in the $F=0$ state, it remains dark, allowing the quantum state of the atomic qubit to be distinguished with an efficiency of about $98 \%$ [23]. It is important to note that unlike single photon detection, every attempt to detect the state of an ion gives a result. The efficiency quoted here is the probability that this result is correct.

To verify that the emitted photon is indeed entangled with the ion, we temporarily add an additional half-wave or quarter-wave plate and a polarizer (see Fig. 1h). In this case the ion manipulation and detection sequence is triggered on the detection of a single photon.

Quantum state tomography is performed for full characterization of the the state of the ion-photon system 22, 27]. We chose to measure both the ion and the photon states in the $\left\{\sigma_{j} \sigma_{k} ; j, k=x, y, z\right\}$ bases. Each measurement is integrated for 100 seconds with an average rate of about 25 ion-photon entanglement events per second. The state tomography algorithm follows the maximum likehood estimation technique described in [28], with the result shown in Fig. 3 From the reconstructed density matrix, we calculate the entanglement fidelity $F_{i p}=0.925 \pm 0.003$, concurrence $C_{i p}=0.861 \pm 0.006$ and entanglement of formation $E_{\text {Fip }}=0.805 \pm 0.008$. We also have measured a Bell inequality parameter $S$ for our
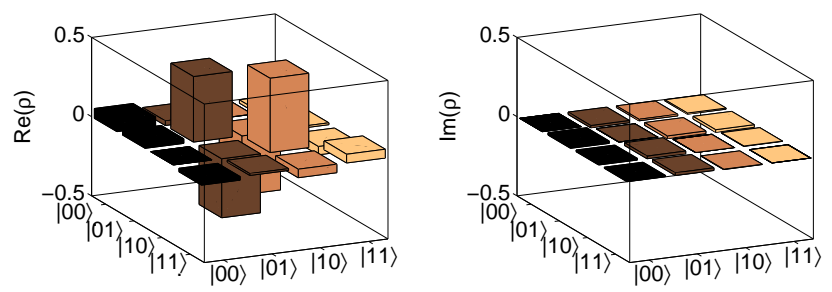

FIG. 4: Real (left) and imaginary (right) parts of reconstructed density matrix for the system of two entangled ions. Results are based on 2121 coincidence events.

ion-photon system [10]. The result of the measurement $(S=2.54 \pm 0.02>2)$ clearly violates the Clauser-HorneSimony-Holt version of the Bell inequality [29], described below.

This high measured entanglement fidelity between a single ion and a single photon allows us to establish entanglement between two remote ions in violation of a Bell inequality. With two ions simultaneously excited, the photoelectric pulses from the PMTs on both output ports of a beamsplitter arriving within a \pm 25 ns coincidence window indicate a successful entanglement event. Following the second photoelectric pulse from the PMTs, the states of both ions are rotated and detected as described above.

To verify the Bell inequality violation, we keep the phase $\phi$ for both ions at 0 and vary $\theta$. Following ClauserHorne-Simony-Holt (CHSH) 29], we calculate the correlation function $E\left(\theta_{a}, \theta_{b}\right)$ given by

$$
E\left(\theta_{a}, \theta_{b}\right)=p\left(\theta_{a}, \theta_{b}\right)+p\left(\theta_{a}^{\perp}, \theta_{b}^{\perp}\right)-p\left(\theta_{a}^{\perp}, \theta_{b}\right)-p\left(\theta_{a}, \theta_{b}^{\perp}\right),
$$

where $p\left(\theta_{a}, \theta_{b}\right)$ is the probability to find ion $(a)$ in the state $\cos \left(\theta_{a} / 2\right)|1,1\rangle+\sin \left(\theta_{a} / 2\right)|1,-1\rangle$ and ion $(b)$ in the state $\cos \left(\theta_{b} / 2\right)|1,1\rangle+\sin \left(\theta_{b} / 2\right)|1,-1\rangle, \theta_{a, b}^{\perp}=\theta_{a, b}+\pi$.

The CHSH version of a Bell inequality states that for all local realistic theories

$$
S=\left|E\left(\theta_{a}, \theta_{b}\right)+E\left(\theta_{a}^{\prime}, \theta_{b}\right)\right|+\left|E\left(\theta_{a}, \theta_{b}^{\prime}\right)-E\left(\theta_{a}^{\prime}, \theta_{b}^{\prime}\right)\right| \leq 2 .
$$

The result of the Bell inequality measurement is given in Table I, based on 2276 coincidence events. On average, we observed 1 entanglement event per 39 sec. The result $S=2.22 \pm 0.07$ represents a Bell inequality violation by more than three standard deviations. Since every heralded entanglement event is followed by the measurement of the qubit states, the Bell inequality violation is observed with the detection loophole closed.

We also performed state tomography for the entangled state of the two ions. As in the ion-photon case, ion measurements were performed in the $\left\{\sigma_{j} \sigma_{k} ; j, k=x, y, z\right\}$ bases, with the result shown in Fig. 4. From this density matrix we estimate the entanglement fidelity $F_{i i}=$ $0.813 \pm 0.015$, concurrence $C_{i i}=0.64 \pm 0.03$, and entanglement of formation $E_{F i i}=0.52 \pm 0.04$. 
TABLE I: Measured correlation function $E\left(\theta_{a}, \theta_{b}\right)$ and CHSH parameter $S$ for the ion-ion state. Errors are based on the statistics of the photon counting events.

\begin{tabular}{ccc}
\hline \hline$\theta_{a}$ & $\theta_{b}$ & $E\left(\theta_{a}, \theta_{b}\right)$ \\
\hline$\pi / 2$ & $\pi / 4$ & $-0.518 \pm 0.036$ \\
$\pi / 2$ & $3 \pi / 4$ & $-0.546 \pm 0.034$ \\
0 & $\pi / 4$ & $-0.581 \pm 0.034$ \\
0 & $3 \pi / 4$ & $0.573 \pm 0.035$ \\
& & $S=2.22 \pm 0.07$ \\
\hline \hline
\end{tabular}

The ion-photon entanglement fidelity is mainly limited by the detection efficiency of the ion state $3 \%$ decrease of fidelity), a fluctuating ambient magnetic field that causes ion dephasing $(2 \%)$, imperfect compensation of the stress-induced birefringence in the viewports of our vacuum chambers and imperfections in polarization control for light propagating through the fibers (1\%), decay of the ion to the $m_{F}=0$ state due to nonzero solid angle of the photon collection (1\%), and PMTs dark counts $(<0.5 \%)$. In addition, interference contrast of the interferometer contributes to the reduced entanglement fidelity of two ions $(9 \pm 3) \%$ compared to the ideal interference between the photons $F_{i i}^{i d e a l}=86 \%$, calculated from the reconstructed ion-photon state. The 13 fold improvement in the entanglement generation rate compared to our previous experiment [20] is due to a different excitation scheme that allows the transfer of all the population to the excited state and a different direction for photon collection with respect to the applied magnetic field that does not require polarization filtering of the collected photon.

Here we have successfully extended the separation between entangled particles by more than 5 orders of magnitude, as compared to the previous Bell inequality test performed with the detection loophole closed [7]. However, a much larger separation between the ions or a shorter detection time are necessary for a loophole-free test of a Bell inequality. For example, a realistic $50 \mu \mathrm{s}$ detection time will require $15 \mathrm{~km}$ separation between the ions [10]. This may be difficult, since absorption of $369.5 \mathrm{~nm}$ light in the optical fiber is relatively large $(\simeq 0.2 \mathrm{~dB} / \mathrm{m})$. Therefore, frequency conversion [30] or free space light transmission may be an alternative solution. Entanglement over larger distances could also be generated using the quantum repeater protocol [31]. Remote entanglement of atomic qubits is also an important step towards the implementation of scalable quantum information processing and scalable quantum communication networks.

This work is supported by the NSA and the IARPA under Army Research Office contract, and the NSF Physics at the Information Frontier Program.

* Electronic address: dmats@umd.edu

$\dagger$ Present address: Max-Planck-Institut für Quantenoptik, Hans-Kopfermann-Str. 1, D-85748 Garching, Germany

[1] J. S. Bell, Physics 1, 195 (1964).

[2] S. J. Freedman and J. F. Clauser, Phys. Rev. Lett. 28, 938 (1972).

[3] A. Aspect, J. Dalibard, and G. Roger, Phys. Rev. Lett. 49, 1804 (1982).

[4] G. Weihs et. al, Phys. Rev. Lett. 81, 5039 (1998).

[5] M. Lamehi-Rachti and W. Mittig, Phys. Rev. D 14, 2543 (1976).

[6] A. Bramon, and M. Nowakowski, Phys. Rev. Lett. 83, 1 (1999).

[7] M. A. Rowe et al., Nature 409, 791 (2001).

[8] Y. Hasegawa et al., Nature 425, 45 (2003).

[9] A. Go, J. of Mod. Opt. 51, 938, (2004).

[10] D. L. Moehring et al., Phys. Rev. Lett. 93, 090410, (2004).

[11] D. N. Matsukevich et al., Phys. Rev. Lett. 95, 040405 (2005).

[12] D. N. Matsukevich et al., Phys. Rev. Lett. 96, 030405 (2006).

[13] C.-W. Chou, et al., Science 316, 1316 (2007).

[14] N. Gisin, G. R. W. Tittel, and H. Zbinden, Rev. Mod. Phys. 74, 145 (2002).

[15] P. G. Kwiat, P. H. Eberhard, A. M. Steinberg, and R. Y. Chiao, Phys. Rev. A 49, 3209 (1994).

[16] S. F. Huelga, M. Ferrero, and E. Santos, Phys. Rev. A 51, 5008 (1995).

[17] E. S. Fry, T. Walther, and S. Li, Phys. Rev. A 52, 4381 (1995).

[18] C. Simon and W. T. M. Irvine, Phys. Rev. Lett. 91, 110405, (2003).

[19] R. García-Patrón et al., Phys. Rev. Lett. 93, 130409 (2004).

[20] D. L. Moehring et al., Nature 449, 68 (2007).

[21] D. L. Moehring et al., J. Opt. Soc. Am. B 24, 300 (2007).

[22] J. Volz, et al., Phys. Rev. Lett. 96, 030404, (2006).

[23] S. Olmschenk, et al., Phys. Rev. A 76, 052314 (2007).

[24] P. Maunz, et al., Nature Physics 3, 538 (2007).

[25] C. K. Hong, Z. Y. Ou, and L. Mandel, Phys. Rev. Lett. 59, 2044 (1987); Y. H. Shih and C. O. Alley, Phys. Rev. Lett. 61, 2921 (1988).

[26] B. B. Blinov, D. L. Moehring, L.-M. Duan, and C. Monroe, Nature 428, 153 (2004).

[27] T. Wilk, S. C. Webster, A. Kuhn, and G. Rempe, Science 317, 488 (2007).

[28] D. F. V. James, P. G. Kwiat, W. J. Munro, and A. G. White, Phys. Rev. A 64, 052312 (2001).

[29] J. F. Clauser, M. A. Horne, A. Shimony, and R. A. Holt, Phys. Rev. Lett. 23, 880 (1969).

[30] S. Tanzilli et al., Nature 437, 116 (2005).

[31] H.-J. Briegel et al., Phys. Rev. Lett. 81, 5932 (1998); W. Dür et al., Phys. Rev. A 59, 169 (1999); L.-M. Duan, M. D. Lukin, J. I. Cirac, and P. Zoller, Nature 414, 413 (2001). 